\title{
Encontro do Idoso Saudável: um relato de experiência sobre educação em saúde interprofissional
}

\author{
Healthy Elderly Meeting: an experience report about interprofessional health education
}

Encuentro de Ancianos Saludables: informe de experiencia sobre educación para la salud interprofesional

Luma Lobato Pinheiro ${ }^{1 *}$, Aline dos Reis Ribeiro ${ }^{1}$, Bruno Souza dos Santos ${ }^{1}$, Elana Braga Costa da Silva ${ }^{1}$, Taize de Nazaré da Silva Oliveira ${ }^{1}$.

\section{RESUMO}

Objetivo: Relatar a experiência dos integrantes do PET-Saúde/Interprofissionalidade no desenvolvimento de ações em saúde voltadas para o público da terceira idade, por meio do "Encontro do Idoso Saudável". Relato da experiência: Trata-se de um relato de experiência sobre o "Encontro do Idoso Saudável", realizado no período de Outubro a Dezembro de 2019 em uma Unidade Básica de Saúde (UBS) localizada no Estado do Pará. O encontro tinha como finalidade principal, a promoção do envelhecimento ativo aos indivíduos da terceira idade, incentivando uma maior interação entre os idosos, através de grupos de convivência; abordando temas de relevância para a faixa etária em questão, tendo como público alvo os usuários da UBS em que o programa atuava. Considerações finais: A interprofissionalidade é importante no contexto acadêmico e profissional, e promover educação em saúde de maneira interprofissional à comunidadepropicia melhor compreensão sobre a relevâcia da prevenção edo tratamento de doenças, assim como a experiência do trabalho integrado, para um mesmo fim.

Palavras-chave: Comunicação interdisciplinar, Educação em saúde, Saúde do idoso.

\begin{abstract}
Objective: Report the experience of the members of PET-Saúde/Interprofessional in the development of health actions aimed at the elderly, through the "Encounter of the Healthy Elderly". Experience report: This is an experience report about the "Meeting of the Healthy Elderly", held from October to December of 2019 in a Basic Health Unit (BHU), located in the State of Pará. The main purpose of the meeting was to promote active aging to elderly individuals, encouraging greater interaction among the elderly, through social groups; approaching topics of relevance for the age group in question, having as target audience users of the UBS which the program worked. Final considerations: Interprofessionality is important in the academic and professional context, and promoting health education in an interprofessional way to the community provides a better understanding of the importance of disease prevention and treatment, as well as the experience of integrated work, for the same purpose.
\end{abstract}

Keywords: Interdisciplinary communication, Health education, Elderly health.

\section{RESUMEN}

Objetivo: Conocer la experiencia de los integrantes de PET-Saúde/Interprofessionalidade en el desarrollo de acciones de salud dirigidas a las personas mayores, a través del "Encuentro del Anciano Sano". Informe de experiencia: Este es un informe de experiencia sobre el "Encuentro de Ancianos Saludables", realizado de octubre a diciembre de 2019 en la Unidad Básica de Salud (UBS), ubicado en el estado de Pará. El principal

${ }^{1}$ Centro Universitário do Estado do Pará (CESUPA), Belém - PA. *E-mail: lumalp@hotmail.com 
objetivo del encuentro fue promover el envejecimiento activo de las personas mayores, fomentando una mayor interacción entre las personas mayores, a través de grupos sociales; abordar temas de relevancia para el grupo de edad en cuestión, teniendo como público objetivo a los usuarios de la UBS en la que se desarrolló el programa. Consideraciones finales: La interprofesionalidad es importante en el contexto académico y profesional, y promover la educación en salud de manera interprofesional a la comunidad brinda una mejor comprensión de la relevancia de la prevención y el tratamiento de enfermedades, así como la experiencia del trabajo integrado, con el mismo propósito.

Palabras clave: Comunicación interdisciplinar, Educación para la salud, Salud del anciano.

\section{INTRODUÇÃO}

A contemporaneidade encontra-se marcada por grandes mudanças nos setores político, científico, cultural, tecnológico e econômico com crescente complexidade e fragmentação da assistência à saúde. Dessa maneira, a prática Interprofissional ganhou projeção e reconhecimento na promoção à saúde de forma mais efetiva, segura e integral (ALMEIDA RGS, et al., 2019; ESCALDA P e PARREIRA CMSF, 2018).

Ao constatar as limitações em alcançar a integralidade do cuidado,por meio de práticas isoladas e fragmentadas, o conceito de Educação Interprofissional (EIP), é apresentado como uma maneira dereorganizar o processodetrabalho emsaúde, no entanto, este, ainda passa por diversos percalços, no que se refere àsua implantação nos perfis curriculares dos cursos de graduação em saúde no País (RIOS DRS, et al., 2020).

O Programa de Educação pelo Trabalho para a Saúde (PET-Saúde) surgiu para auxiliar a formação dos acadêmicos de saúde para atender ao perfil socioepidemiológico da população brasileira e tem como base a integração ensino-serviço-comunidade (FARIAS BC e NOROLR, 2017).

Em 2018 foi lançado o PET-Saúde/Interprofissionalidade,que envolve atores do Sistema Único de Saúde (SUS): profissionaisde saúde, gestoreseusuários; bem como da comunidade acadêmica, como professores e estudantes. Considera ações a serem desenvolvidas na Atenção Primária à Saúde (APS) para a qualificação dos futuros profissionais que, numa prática colaborativa, poderão obter respostas mais efetivas na melhoria da atenção à saúde (STARFIELD B, 2002; REEVES S, et al., 2016; BATISTA NA, et al., 2018; BRASIL, 2018; PEREIRA MF, 2018).

Para alcançamos resultados satisfatórios com a prática da educação em saúde, é importante que haja uma boa interação entre os profissionais da área que valorizem a promoção e prevenção em saúde; dos gestores, propiciando o devido suporte aos profissionais e da população, desse modo, ocorrerá a construção de conhecimentos e o aumento de sua autonomia nos cuidados, individual e coletivamente. Segundo a Organização Mundial da Saúde (OMS) o termo "envelhecimento ativo" e expressa o processo de conquista dessa visão sobre o envelhecer (FALKENBERG MB, et al., 2014; OMS, 2005).

Na unidade básica de saúde em que o grupo tutorial do PET-Saúde em questão, estava implantado, notouse que grande parte dos usuários eram idosos. Dessa forma, observou-se uma necessidade de planejar educações em saúde voltada para esse grupo, de forma a promover informações e socialização entre os idosos, buscando atender às suas necessidades específicas.

\section{RELATO DE EXPERIENCIA}

Esse relato foi vivenciado na Unidade Básica de Saúde (UBS) localizada em um municipio do estado do Pará, na qual um grupo tutorial do PET-Saúde estava inserido; composto por alunas, preceptores e tutores, vínculados ao Centro Universitário do Estado Pará (CESUPA). Entre os meses de Outubro e Dezembro de 2019, foram desenvolvidas ações de educação em saúde, por meio do "Encontro do Idoso Saudável" cujo objetivo foi promover aos indivíduos da terceira idade um envelhecimento ativo, incentivando uma maior interação entre os idosos por meio de grupos de convivência, abordando temas de relevância para esta faixa etária, tendo como público alvo os usuários da UBS em que o programa atuava. 
A seleção dos temas a serem abordados e a elaboração das ações, eram feitos em reuniões do grupo tutorial constituído por cinco alunas bolsistas do PET, de cursos da área da saúde distintos, tais quais: Odontologia, medicina, enfermagem, fisioterapia e nutrição; Preceptores: profissionais da saúde que trabalhavam na UBS e orientavam as ações, sendo estes: um médico, uma enfermeira e uma odontóloga; e Tutores: docentes da área da saúde do CESUPA, responsáveis pelo monitoramento e dar o suporte técnicocientífico às ações.

Os encontros eram realizados em uma quadra poliesportiva pública, localizada próxima à UBS do bairro, pelo período da manhã. As alunas ficavam encarregadas pela apresentação das temáticas no dia proposto, enquanto que os Agentes Comunitários de Saúde (ACS'S) da UBS eram os responsáveis pela divulgação dos encontros, sendo esta feita de maneira verbal no momento em que os idosos estavam presentes na unidade ou através de panfletos digitais, disseminados por meio de um aplicativo de menssagens instantâneas.

O primeiro encontro ocorreu no dia 26 de outubro de 2019, às 8 horas da manhã. Os temas propostos foram "prevenção de quedas em idosos" e a "importância da atividade física na terceira idade", 35 idosos participaram nesse dia. Iniciou-se com a aferição de pressão arterial e medição da glicemia, haja vista que a verificação desses índices devem ser feitas de modo contínuo, por trata-se de longevos.

Em seguida foi oferecido um café da manhã com caráter nutritivo com frutas e chá, a posteriori, os temas propostos foram abordados por meio de "rodas de conversa", o que induziu uma melhor interação do público com as alunas e preceptores do PET-Saúde, os participantes contribuíram com seus conhecimentos e experiências acerca dos temas, e uma dinâmica de "perguntas e respostas" foi introduzida com a intenção de criar um ambiente confortável para tirar dúvidas sobre os temas, o que estimulou entre os idosos uma maior interação.

Objetos como tapetes, sapatos de salto e figuras foram utilizados para ilustrar os fatores extrínsecos relacionados a queda em idosos; Exemplos de exercícios físicos e alongamentos foram apresentados pela acadêmica de fisioterapia e um educador físico aos longevos para que estes pudessem reproduzir em sua residência.

No segundo "Encontro do idoso saudável", realizado no dia 23/11/19 os temas de escolha para serem apresentados foram: "Doença periodontal", "cuidados com a prótese dental" e "hábitos saudáveis alimentares com ênfase nos alimentos regionais". Iniciou- se o encontro com a temática da saúde bucal do idoso. Em um primeiro momento, foi feita uma orientação de higiene bucal utilizando-se de macro modelos de "boca" e escova de dente, demonstrou-se as corretas técnicas de escovação, passagem do fio dental e outros pontos relevantes ao assunto.

Após a demonstração, apresentou-se aos idosos o tema: "Doença periodontal", que foi escolhido pelo fato de essa ser uma das patologias bucais que mais acometem a população idosa, levando em consideração que essa população tende a sofrer de doenças sistêmicas e fazer o uso de medicamentos, tais aspectos se relacionam com a doença periodontal (ROCHA EF, et al., 2019).

Em um momento posterior abordamos o tema: "Cuidados com a prótese dental". Foi feita a distribuição de panfletos que continham as informações referentes ao tema, para que os idosos pudessem acompanhar no momento da educação em saúde e após o término do encontro tivessem as informações disponíveis de maneira acessível. Abriu-se um espaço para a retirada de dúvidas, no qual foi possível notar a grande participação dos idosos e o interesse a respeito dos temas abordados.

Dando continuidade ao encontro, as acadêmicas do PET-Saúde introduziram o último tema do dia: "Hábitos saudáveis alimentares com ênfase nos alimentos regionais" na forma de "roda de conversa". Optouse por apresentar esse tema de uma maneira mais lúdica. Foi dado a cada um dos idosos, imagens de alimentos variados e um prato descartável, em seguida orientou-se a eles comporem o prato com as imagens dos alimentos que mais eram presentes no seu dia a dia.

A acadêmica de nutrição foi a responsável por dialogar com os participantes sobre a alimentação, dando orientações de como obter hábitos alimentares mais saudáveis, mostrando por meio das imagens quais seriam os alimentos que deveriam estar presentes com maior frequência na dieta, enfatizando o uso de 
alimentos típicos da região, os quais os idosos apresentam um maior contato e podem adquirir com facilidade por um valor mais acessível, além de relacionar a comida saudável com a higiene bucal, que estão intimamente conectadas; e assim concluiu-se o segundo encontro.

O terceiro momento de atividades do "Encontro do Idoso Saudável" foi realizado no dia 14/12/19 e foram abordados dois temas principais, intitulados: "Menopausa e suas repercussões", voltado principalmente para o público feminino da terceira idade, e "Incontinência urinária", considerada uma queixa comum no dia a dia dos idosos.

No início do encontro, foi realizada uma atividade lúdica para explanação do tema "Menopausa", com perguntas e respostas realizadas pelas alunas do grupo tutorial às idosas participantes, quando puderam questionar fatos importantes sobre tal assunto, como idade mais comum de acometimento e principais sintomas, além da repercussão deste marco na saúde das mulheres idosas. As participantes da atividade respondiam com "verdadeiro" ou "falso" as perguntas realizadas, a partir das quais puderam sanar suas principais dúvidas acerca do tema, além de adquirir novos conhecimentos com uma pequena roda de conversa após cada pergunta sobre o assunto.

Em um segundo momento da atividade, foi abordado o tema "Incontinência Urinária". As acadêmicas de enfermagem e fisioterapia realizaram uma dinâmica com os idosos, explanando a fisiopatologia da Incontinência utilizando recursos para explanar o tema didaticamente, com uma linguagem compreensível à população. Balões de festa foram preenchidos com água e foram utilizados para demonstrar o reflexo de micção, e como o órgão se comporta no contexto da Incontinência Urinária. Além disso, as alunas explanaram quais os principais sinais e sintomas da afecção, momento este que os idosos também puderam compartilhar suas dúvidas com o Grupo Tutorial.

Através das atividades de extensão realizadas, foi possível promover educação em saúde aos idosos, além de permitir a troca de saberes e experiências entre os participantes do PET, assim como foi possível obter um conhecimento maior acerca dos principais agravos que acometem a população longeva residente do conjunto Julia Seffer, permitindo com que os alunos, preceptores e tutores pudessem planejar ações com um maior enfoque aos anseios da população. As atividades, não só beneficiam a própria população, como também permitem a ampliação dos conhecimentos científicos, já que exigem preparo por parte dos estudantes acerca de temas da própria área de atuação e acerca de outras áreas, permitindo uma maior vivencia da interprofissionalidade.

Os idosos receberam as atividades de maneira positiva, sendo bastante participativos em todos os encontros realizados, quando puderam além de adquirir novos conhecimentos acerca de temas de seus interesses, aumentar o vínculo com profissionais das diferentes áreas de atuação participantes do projeto PET-Saúde. Foram relatados pela própria população que as atividades de educação em saúde promoveram ampliação do saber coletivo, além de promove-los uma maior autonomia acerca de seus agravos, possibilitando um maior entendimento de si próprio e da importância do conhecimento adquirido durante os encontros no contexto saúde-doença.

\section{DISCUSSÃO}

Ao longo dos anos, os serviços de atenção e cuidado à saúde, priorizaram o diagnóstico e o tratamento de doenças, sendo centralizado na ótica terapêutica, em detrimento do estudo holístico do indivíduo e seus aspectos psicossociais. Para que haja uma organização adequada desse trabalho que tem como enfoque visualizar o cidadão de maneira integral, é necessário haver "estabelecimento vínculos, responsabilização e participação popular", a fim de gerar ações proveitosas de forma igualitária, que atuem como mediador entre os conflitos existentes na comunidade, levando em consideração toda sua diversidade (MELO RH, et al., 2016).

Nesse contexto é de suma importância a educação em saúde continuada aumentando a interação entre profissionais e população com intuito de provocar mudanças que gerem um impacto no processo saúde doença que acometem diversos setores da comunidade no qual o projeto "PET-Saúde interprofissionalidade" está inserido (PINHEIRO LL, et al., 2021). 
Foi observada a necessidade de um maior número de ações em saúde voltados para a população da terceira idade, tendo em vista que formam uma parcela significativa na comunidade em questão, como também são atores muito importantes no contexto saúde-família-comunidade, visto que são muito acometidos por doenças crônico-degenerativas e necessitam de cuidados longitudinais (PEREIRA RP, et al., 2016; CYRINO RS, et al., 2016).

As informações sobre autocuidado são importantes, pois a doença ou lesão de órgão alvo podem alterar o funcionamento dos sistemas do corpo humano, trazendo necessidades temporárias ou permanentes (SILVA IJ, et al., 2009). Constatou-se que a associação da educação em saúde e a prevenção de doenças crônicodegenerativas na população, abordada nas ações interdisciplinares, é de grande valia tanto para a população quanto para os membros integrantes do PET-Saúde, pois possibilita a interação e a "troca de conhecimento" entre ambos, de uma maneira clara e sucinta. (CAMPOS CG, et al., 2018; CARDOSO RR, et al., 2016).

Ao iniciar as práticas da interprofissionalidade, estas tornaram-se um desafio para os alunos, preceptores e tutores. Isto pode ser atribuído a ausência de qualificação nas instituições durante o processo de preparação e formação do discente e docente e está relacionado ao fato de que: apesar das transformações históricas do Serviço Social, destacando ainda a crescente demanda nos estágios curriculares ou não na área da saúde, considera-se incipiente o status da educação interprofissional e atividades voltadas a práticas colaborativas, sobretudo em disciplinas obrigatórias durante a formação dos acadêmicos (MARQUES GB, et al., 2020). Mudanças nos cursos de graduação e no serviço de saúde pautadas na experiência do PET-Saúde são intrincadas e de longo prazo, envolvendo entraves em diferentes níveis, distanciados da realidade dos participantes do Programa (LOURENÇO AE, et al., 2017).

Durante o processo de execução do projeto, os integrantes se depararam com alguns obstáculos a serem superados para alcançarem os objetivos propostos, dentre eles, se ressalta a incompatibilidade de horários entre os integrantes do grupo tutorial, assim como a dificuldade de conciliação da grade curricular dos estudantes para o desenvolvimento das atividades interdisciplinares em campo.

No intuito de reparar essas limitações, algumas medidas resolutivas foram adotadas pelo grupo, como por exemplo: Para a realização do "Encontro do Idoso Saudável" se deu preferência pelos sábados durante o turno da manhã, sendo este o dia em que a maioria das alunas e dos preceptores apresentavam disponibilidade para o desenvolvimento das atividades. Além disso, o público de idosos se apresentava com maior frequência nas ações realizadas neste período estabelecido.

De maneira convergente, pesquisas referentes ao Pet-Saúde de regiões diversificadas do Brasil apresentaram a indisponibilidade e as limitações para a articulação de horários entre os integrantes do PET, como grandes desafios para se atingir o progresso do programa (BELLINI LK, et al., 2020; MAGNAGO C, et al., 2019; MIRA QL, et al., 2020; VIEIRA VB, et al., 2020).

A interprofissionalidade é de suma importância no contexto acadêmico e profissional. Educar de forma interprofissional ainda é um grande desafio para os profissionais da saúde, porém permite o desenvolvimento de uma visão mais ampla sobre os métodos de promoção, prevenção e recuperação da saúde de cada indivíduo. Promover educação em saúde de maneira interprofissional propicia à comunidade, uma melhor compreensão sobre a importância de prevenir e tratar doenças em todas as fases da vida, bem como a experiência do trabalho integrado, para um mesmo fim.

\section{REFERÊNCIAS}

1. ALMEIDA RGS, et al. A interface entre o PET-Saúde/Interprofissionalidade e a Política Nacional deEducação Permanente em Saúde. Saúde Debate, 2019; 43(1): 97-105.

2. BATISTA NA, et al. Educação interprofissional na formação em Saúde a experiência da Universidade Federal de São Paulo, campus Baixada Santista. Interface, 2018; 22(2): 1705-15.

3. BELLINI LK, et al. A inserção do curso de psicologia no Pet-Saúde interprofissionalidade: um relato de experiência. Saberes Plurais: Educação na Saúde, 2020; 4(1): 41-52.

4. BRASIL. Ministério da Saúde. Construindo caminhos possíveis para a Educação Interprofissional em Saúde nas Instituições de Ensino Superior do Brasil. Brasília, DF: Ministério da Saúde. 2018. Disponível 
em:https://www.educacioninterprofesional.org/pt/construindo-caminhos-possiveis-para-educacao-interprofissionalem-saude-nas-instituicoes-de-ensino. Acesso em: 14 de junho de 2021.

5. CAMPOS CG, et al. PET-Saúde/GRADUASUS da enfermagem: Acolhimento dos usuários de uma unidade de saúde da família. Revista Conexão UEPG, 2019; 15(1): 092-097.

6. CARDOSO RR, et al. Promovendo educação em saúde na sala de espera das unidades de saúde: relato de experiência. Revista Norte Mineira de Enfermagem, 2016; 5(1): 97-104.

7. CYRINO RS, et al. Atividades lúdicas como estratégia de educação em saúde com idosos. Revista Ciência em Extensão, 2016; 12(3): 154-163.

8. ESCALDA P, PARREIRA CMSF. Dimensões do trabalho interprofissional e práticas colaborativas desenvolvidas em uma unidade de básica de saúde, por equipe de Saúde da Família. Interface, 2018; 22(2) 1717-27.

9. FALKENBERG MB, et al. Educação em saúde e educação na saúde: conceitos e implicações para a saúde coletiva. Ciência \&amp; Saúde Coletiva, 2014; 19: 847-852.

10. FARIAS BC, NORO LR. PET-Saúde como indutor da formação profissional para o Sistema Único de Saúde. Ciência \&amp; Saúde Coletiva, 2017; 22(3): 997-1004.

11. LOURENÇO AE, et al. Programa de Educação pelo Trabalho para a Saúde (PET-Saúde) e a formação do nutricionista num campus de interiorização. Demetra: Alimentação, Nutrição e Saúde, 2017; 12(1): 41-58.

12. MAGNAGO C, et al. PET-Saúde/GraduaSUS na visão de atores do serviço e do ensino: contribuições, limites e sugestões. Saúde Debate, 2019; 43(1): 24-39.

13. MARQUES GB, et al. Educação interprofissional na graduação: Os desafios sob a ótica dos acadêmicos no PETSaúde em Manaus. Brazilian Journal of Development, 2020; 6(6): 3680436807.

14. MELO RH, et al. Roda de conversa: uma articulação solidária entre ensino, serviço e comunidade. Revista Brasileira de Educação Médica, 2016; 40(2): 301-309.

15. MIRA QL, et al. Reorientação da formação profissional em saúde: o que nos dizem os relatórios finais dos PET-SAÚDE e PRÓ-PET-SAÚDE?. Revista de APS, 2020; 23(1): 175-194.

16. PEREIRA RP, et al. A avaliação global da pessoa idosa como instrumento de educação médica: relato de experiência. Revista Brasileira de Educação Médica, 2016; 40(2): 314-320.

17. PEREIRA, MF. Interprofissionalidade e saúde: conexões e fronteiras em transformação. Interface, 2018, 22(2): 17536.

18. REEVES S, et al. A BEME systematic review of the effects of interprofessional education: BEME Guide No. 39. Med Teach, 2016; 38(7): 656-68.

19. RIOS DRS, et al. Diálogos interprofissionais e interdisciplinares na prática extensionista: o caminho para a inserção do conceito ampliado de saúde na formação acadêmica. Interface, 2020; (23): e180080.

20. ROCHA EF, et al. Envelhecimento humano e desenvolvimento da doença periodontal. Revista Eletrônica Acervo Saúde, 2019; (26): e775.

21. SILVA IJ, et al. Cuidado, autocuidado e cuidado de si: uma compreensão paradigmática para o cuidado de enfermagem. Rev. esc. enferm, 2009 ; 43(3): 697-703.

22. STARFIELD B. Atenção primária: equilíbrio entre necessidades de saúde, serviços e tecnologia. 2002.

23. VIEIRA VB, et al. O Programa de Educação pelo Trabalho para a Saúde (PETSAÚDE) sob a ótica dos preceptores. Revista Educação e Emancipação, 2020; 13(1): 202-220.

24. WORLD HEALTH ORGANIZATION (WHO). Envelhecimento ativo uma política de saúde. Brasília: Organização PanAmericana da Saúde, 2005. Disponível em: https://bvsms.saude.gov.br/bvs/publicacoes/envelhecimento_ativo.pdf. Acesso em: 14 de junho de 2021. 